\title{
Código cíclico óptimo y constelaciones rotadas para canales de transmisión con línea eléctrica de bajo voltaje
}

\author{
Optimal cyclic code and rotated constellations for channel \\ of transmission with low voltage electric line \\ Washington Fernández ${ }^{1}$ \\ Recibido 13 de agosto de 2013, aceptado 17 de junio de 2014 \\ Received :August 13, 2013 Accepted: June 17, 2014
}

\begin{abstract}
RESUMEN
En este artículo se estudia el desempeño de la combinación de un código cíclico óptimo concatenado con constelaciones de rotaciones de ángulos bidimensionales (rectangular, circular y hexagonal) de cuatro y dieciséis símbolos. El desempeño se evalúa respecto de la razón de error de bits versus la razón de señal a ruido, los resultados que se obtienen permiten una gran mejora respecto de cuando solo se emplean las constelaciones con rotaciones de ángulos.
\end{abstract}

Palabras clave: Código cíclico, constelaciones bidimensionales, constelaciones rotadas, ruido Middleton, ruido en canales con línea eléctrica de bajo voltaje.

\section{ABSTRACT}

In this article the performance of the concatenation of an optimum cyclic code with two-dimensional angle rotation constellations (rectangular, circular and hexagonal) of four and sixteen symbols is studied. The performance is evaluated with respect to the bit error rate versus signal to noise ratio. The results allow for a great improvement as compared to using the constellations with angle rotations only.

Keywords: Cyclic code, bi-dimensional constellations, rotated constellations, Middleton noise, channel noise with low voltage electric line.

\section{INTRODUCCIÓN}

La línea eléctrica de bajo voltaje como un medio de transmisión de dato digital es muy adversa porque esta no se construyó para transmitir dato, sino energía eléctrica. El ruido presente en la línea eléctrica de bajo voltaje contiene un ruido gaussiano de fondo $\mathrm{y}$ un ruido impulsivo.

Para mejorar el desempeño del sistema de transmisión/ recepción se tiene una variedad de técnica de modulación y codificación.

Por ejemplo, se tiene el trabajo de [1] que estudia las constelaciones que minimiza la probabilidad de error en un canal con ruido clase A. En [2] se dan técnicas para generar y optimizar un conjunto de señales hexagonales. En [3] se presenta el ángulo óptimo para modulaciones 8-PSK y 16-QAM, para lograrlo minimiza una función de costo. En [4] se da una expresión analítica para la rotación óptima de ángulos con el criterio de la distancia euclidiana para cualquier constelación M-PSK.

El ruido presente en la línea eléctrica de bajo voltaje tiene un modelo relativamente simple que incorpora el ruido de fondo y el ruido impulsivo el cual se sugiere en [5] y se conoce como ruido Middleton clase A. Este modelo de ruido corresponde a un proceso aleatorio discreto independiente y distribuido

1 Departamento de Ingeniería Eléctrica y Electrónica. Universidad del Bío-Bío. Avenida Collao 1202. Concepción, Chile. 
idénticamente (i.i.d), cuya función densidad de probabilidad es una infinita suma de densidades gaussianas [6].

El canal de línea eléctrica de bajo voltaje es un canal típico de ruido clase $\mathrm{A}$.

Como el ruido clase A contiene ruido impulsivo, que se manifiesta por una gran amplitud y de corta duración, que afecta a varios bits consecutivos cuando se transmite data digital, los códigos cíclicos han demostrado su eficiencia en canales con ruido de ráfagas, es decir, característico de los canales con ruido impulsivo [7-8], por esta razón que en este artículo se optimiza un código cíclico y se emplean las constelaciones rotadas porque estas tienen muy buen desempeño en canales de líneas eléctricas de acuerdo a [1].

Con relación a la investigación en código cíclico se tiene a [9] que da una forma de construir código cíclico mejor que los códigos cíclicos Reed-Solomon.

En [10] se encuentra un código binario cíclico (145, 32 ), que tiene un grado 28 y exponente 128 , con un polinomio $x^{4}+x^{3}+x^{2}+1$.

En [11] da un algoritmo para corregir errores de ráfaga de códigos cíclicos, el cual se basa en la matriz de chequeo de paridad circulante.

En [12] se encuentran los pesos de distribución de códigos cíclicos irreducibles, los pesos que encuentra varían entre uno y cuatro.

En [13] da un nuevo algoritmo para decodificar código cíclico, utiliza la decodificación algebraica y concluye que se puede utilizar en cualquier tipo de código cíclico.

En [14] una familia de secuencias Kasami generalizada se estudian y a partir de una familia se encuentran los pesos de distribución de los códigos cíclicos.

En [15] se determina la mínima distancia de todos los códigos lineales cíclicos de longitud 151 y encuentra 39 nuevos códigos lineales binarios que tienen como distancia mínima mayor que los códigos previamente considerados.

En [16] se encuentran códigos cíclicos de dos pesos de distribución que no se pueden reducir más.
En [17] se presenta un nuevo algoritmo para codificar códigos cíclicos de gran longitud.

En este artículo se estudia el desempeño de un sistema de transmisión/recepción que se compone de un codificador/decodificador de código cíclico óptimo, rotaciones óptimas de constelaciones bidimensionales: hexagonal, circular y rectangular de cuatro y dieciséis símbolos para un canal con línea eléctrica de bajo voltaje. Los parámetros de confiabilidad son la razón de bits error (BER) versus la razón de señal a ruido (SNR).

La organización del trabajo es la siguiente: en la sección siguiente se analiza el sistema de transmisión/ recepción propuesto. A continuación se dan los resultados en forma de gráficos y se discuten los resultados. Finalmente, se pueden encontrar las conclusiones.

\section{ANÁLISIS DEL SISTEMA DE TRANSMISIÓN/RECEPCIÓN PROPUESTO}

Middleton [5] propone en 1977 un modelo de ruido impulsivo, que está conformado por la suma de ruido blanco y ruido impulsivo. En este modelo, según el ancho de banda del ruido se clasifica en tres clases generales: clase A, clase B y clase $\mathrm{C}$.

Ruido clase A: Interferencia cuyos espectros son iguales o más estrechos que el ancho de banda del receptor.

Ruido clase B: Ruido cuyos espectros son más amplios que el ancho de banda del receptor.

Ruido clase C: Interferencia que incluye las características de clase A y B.

De acuerdo con [5], el ruido presente en las líneas eléctricas es del tipo ruido clase A. La función de densidad de probabilidad (FDP) del ruido de Middleton clase A, normalizada se representa por [1]:

$$
p_{z}(z)=\sum_{m=0}^{\infty} \frac{e^{-A} A^{m}}{m !} \frac{1}{\sqrt{2 \pi \sigma_{m}^{2}}} e^{\frac{|z|^{2}}{2 \sigma_{m}^{2}}}
$$

Donde:

$z$ : Envolvente compleja de ruido.

$\sigma_{m}^{2}:$ Varianza de Middleton. 
$m$ : Número de subcanales de Middleton.

$A$ : Índice impulsivo.

El parámetro $A$ se define por el producto entre la duración promedio de los impulsos y la razón de generación media del ruido impulsivo (cantidad de eventos impulsivos por unidad de tiempo).

La varianza de Middleton se obtiene a partir de [1]:

$$
\sigma_{m}^{2}=\sigma^{2} \frac{\frac{m}{A}+\Gamma}{1+\Gamma}
$$

Donde:

$\Gamma$ : Relación entre las potencias gaussiana e impulsiva, está dada por [1]:

$$
\Gamma=\frac{\sigma_{G}^{2}}{\sigma_{i}^{2}}
$$

Esto significa que el modelo de Middleton se representa por el producto de una distribución Poisson, $P(m ; A)$ y una distribución de Gauss $G\left(z ; \sigma_{m}^{2}\right)$. Esto es:

$$
p_{z}(z)=\sum_{m=0}^{\infty} P(m ; A) G\left(z ; \sigma_{m}^{2}\right)
$$

Donde:

$$
P(m ; A)=\frac{e^{-A} A^{m}}{m !}
$$

$\mathrm{y}$

$$
G\left(z ; \sigma_{m}^{2}\right)=\frac{1}{\sqrt{2 \pi \sigma_{m}^{2}}} e^{-\frac{\left|z^{2}\right|}{2 \sigma_{m}^{2}}}
$$

El modelo de Middleton clase A, consiste de la sumatoria de infinitos ruidos gaussianos, cada uno con distinta potencia de ruido. Aquellos que presentan mayor potencia se denominan impulsivos $\mathrm{y}$ los que tienen una potencia de ruido mucho menor se definen como gaussianos.

A continuación se analizan y se encuentran los ángulos óptimos de rotaciones de las constelaciones bidimensionales rectangular, circular y hexagonal de cuatro y dieciséis símbolos y el código cíclico óptimo.

Una constelación con dimensión $N$ es un conjunto finito de $\mathrm{N}$-uplas o puntos en el espacio dimensional $N$. El tamaño de la constelación $|C|$ es el número de puntos. En una constelación bidimensional se tiene $|C|=2^{b}$ puntos, con $b$ número entero.

Sea una constelación en el plano complejo, es decir, de la forma: $q_{i}= \pm x_{i} \pm y_{i}$, como se muestra en la Figura 1.

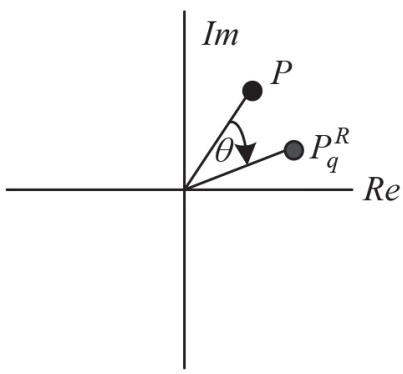

Figura 1. Puntos en el plano complejo.

De acuerdo con la Figura 1, los puntos $P$ y $P_{q}^{R}$ se relacionan con la siguiente transformación:

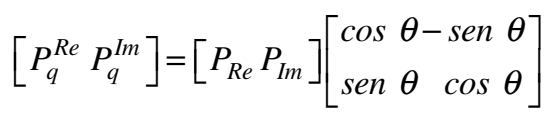

Si el ángulo se mide en el sentido horario los puntos se escriben como:

$$
\begin{aligned}
& x_{i}=a_{i} \cos \theta-b_{i} \operatorname{sen} \theta \\
& y_{i}=a_{i} \operatorname{sen} \theta+b_{i} \cos \theta
\end{aligned}
$$

Donde:

$a_{i}$ : Punto de la constelación sin rotar, eje real.

$b_{i}$ : Punto de la constelación sin rotar, eje imaginario.

Para determinar el símbolo que se transmite, el receptor utiliza la métrica:

$$
d_{i}=\left|R_{1}-x_{i}\right|^{2}+\left|R_{2}-y_{i}\right|^{2}
$$

El detector escoge a favor del símbolo $\hat{s}_{i}=\left(x_{i}, y_{i}\right)$ que minimiza a (10). De [1] se tiene la métrica: 


$$
P_{e}\left(s_{i} \rightarrow \hat{s}_{i}\right) \leq \frac{1}{2}\left(\frac{1}{1+\left|x_{i}-\hat{x}_{i}\right|^{2}}\right)\left(\frac{1}{1+\left|y_{i}-\hat{y}_{i}\right|^{2}}\right)
$$

Donde:

$P_{e}$ : Probabilidad de error de símbolo.

Si se utiliza (8) y (9) y se reemplaza en (11):

$$
P_{e}\left(s_{i} \rightarrow \hat{s}_{i}\right) \geq G_{1} G_{2}
$$

Donde:

$$
\begin{aligned}
G_{1} & =\frac{1}{1+\left|\left(a_{i}-\hat{a}_{i}\right) \cos \theta+\left(b_{i}-\hat{b}_{i}\right) \operatorname{sen} \theta\right|^{2}} \\
G_{2} & =\frac{1}{1+\left|\left(a_{i}-\hat{a}_{i}\right) \operatorname{sen} \theta+\left(b_{i}-\hat{b}_{i}\right) \cos \theta\right|^{2}}
\end{aligned}
$$

Cuando no hay rotación:

$$
\begin{aligned}
\theta & =0 \\
x_{i} & =a_{i} \\
y_{i} & =b_{i}
\end{aligned}
$$

En este caso $G_{1}$ y $G_{2}$ son:

$$
\begin{gathered}
G_{1}=\frac{1}{1+\left|\left(a_{i}-\hat{a}_{i}\right)\right|^{2}} \\
G_{2}=\frac{1}{1+\left|\left(b_{i}-\hat{b}_{i}\right)\right|^{2}}
\end{gathered}
$$

Si se compara (13) con (18) y (14) con (19), se observa que la probabilidad de error depende del ángulo de rotación $\theta$, se debe encontrar el valor de $\theta$ que minimice la probabilidad de error.

Para minimizar la probabilidad de error es equivalente a minimizar la función de costo [1]:

$$
J=\sum_{\hat{s}_{i} \neq s_{i}} P_{e}\left(s_{i} \rightarrow \hat{s}_{i}\right)
$$

La minimización de la función de costo $J$ se realiza numéricamente usando el algoritmo de gradiente dado en [19]:

$$
\theta(k+1)=\theta(k)+u \frac{d J}{d \theta}
$$

Donde:

$u$ : Constante de valor pequeño.

Evaluando (20) y (21) se obtienen los ángulos de rotaciones óptimos para cada una de las constelaciones rectangular, hexagonal y circular para cuatro y dieciséis símbolos, los que se indican en la Tabla 1.

Tabla 1. Ángulos óptimos de rotaciones para las constelaciones rectangular, circular y hexagonal.

\begin{tabular}{|c|c|c|c|c|c|}
\hline $\mathrm{N}=16$ & $X$ & 0,5387 & 0,7149 & $-0,7149$ & $-0,5387$ \\
\hline & $\mathrm{Y}$ & 0,7149 & $-0,5387$ & 0,5387 & $-0,7149$ \\
\hline & $\mathrm{X}$ & 0,3628 & 0,8909 & $-0,8909$ & $-0,3628$ \\
\hline & Y & 1.9666 & $-1,7904$ & 1,7904 & $-1,966$ \\
\hline & $\mathrm{X}$ & 1.7904 & 1,9666 & $-1,9666$ & $-1,7904$ \\
\hline & $\mathrm{Y}$ & 0.8909 & $-0,3628$ & 0,3620 & $-0,8909$ \\
\hline & $\mathrm{X}$ & 1.6145 & 2,1425 & $-2,1425$ & $-1,6145$ \\
\hline & $\mathrm{Y}$ & 2.1425 & $-1,6145$ & 1,6145 & $-2,1425$ \\
\hline
\end{tabular}

\begin{tabular}{|c|c|c|c|}
\hline $\mathbf{N}$ & Rectangular & Circular & Hexagonal \\
\hline 4 & $10^{\circ}$ & $38^{\circ}$ & $25^{\circ}$ \\
\hline 16 & $8^{\circ}$ & $20^{\circ}$ & $18^{\circ}$ \\
\hline
\end{tabular}

Para la constelación rectangular de cuatro símbolos, las magnitudes de los puntos se dan en la Tabla 2 y en la Tabla 3, las magnitudes de la constelación de dieciséis símbolos.

Tabla 2. Magnitudes de los puntos de la constelación rectangular de cuatro símbolos.

\begin{tabular}{|cc|c|c|c|c|}
\hline $\mathrm{N}=4$ & $\mathrm{X}$ & 1,1437 & 1,6334 & $-1,5334$ & $-1,1437$ \\
\hline $\mathrm{Y}$ & 1,6334 & $-1,1437$ & 1,1437 & $-1,4334$ \\
\hline
\end{tabular}

Tabla 3. Magnitudes de los puntos de la constelación rectangular de dieciséis símbolos.

Para la constelación circular de cuatro símbolos, las magnitudes de los puntos se dan en la Tabla 4 y en la Tabla 5, las magnitudes de la constelación de dieciséis símbolos. 
Tabla 4. Magnitudes de los puntos de la constelación circular de cuatro símbolos.

\begin{tabular}{|cc|c|c|c|c|}
\hline $\mathrm{N}=4$ & $\mathrm{X}$ & 1,1111 & 0,8681 & $-1,1111$ & $-0,8681$ \\
\hline & $\mathrm{Y}$ & 0,8681 & $-1,1111$ & $-0,8681$ & 1,1111 \\
\hline
\end{tabular}

Tabla 5. Magnitudes de los puntos de la constelación circular de dieciséis símbolos.

\begin{tabular}{|cc|c|c|c|c|}
\hline $\mathrm{N}=16$ & $\mathrm{X}$ & 0,5482 & $-0,1369$ & $-0,6295$ & $-0,2575$ \\
\hline $\mathrm{Y}$ & 0,3665 & 0,6219 & 0,0661 & $-0,5782$ \\
\hline $\mathrm{X}$ & $-1,657$ & $-1,8931$ & $-1,5276$ & $-1,7826$ \\
\hline $\mathrm{Y}$ & 0,9223 & $-0,1203$ & $-1,1424$ & $-0,6488$ \\
\hline $\mathrm{X}$ & 0,4704 & 1,1488 & 0,1503 & $-0,5922$ \\
\hline $\mathrm{Y}$ & $-0,423$ & 1,5095 & 1,8910 & 0,8378 \\
\hline $\mathrm{X}$ & $-0,677$ & 0,0849 & 1,3306 & 1,8503 \\
\hline $\mathrm{Y}$ & $-1,772$ & -10224 & $-1,3519$ & $-0,4179$ \\
\hline
\end{tabular}

Para la constelación hexagonal de cuatro símbolos, las magnitudes de los puntos se dan en la Tabla 6 y en la Tabla 7, las magnitudes de la constelación de dieciséis símbolos.

Tabla 6. Magnitudes de los puntos de la constelación hexagonal de cuatro símbolos.

\begin{tabular}{|cc|c|c|c|c|}
\hline $\mathrm{N}=4$ & $\mathrm{X}$ & $-0,5959$ & 0,5737 & $-1,2779$ & 0,5737 \\
\hline & $\mathrm{Y}$ & 1,2779 & 0,2675 & $-0,5959$ & $-0,2675$ \\
\hline
\end{tabular}

Tabla 7. Magnitudes de los puntos de la constelación hexagonal de dieciséis símbolos.

\begin{tabular}{|cc|c|c|c|c|}
\hline $\mathrm{N}=16$ & $\mathrm{X}$ & $-0,5862$ & $-1,1873$ & $-1,2925$ & $-0,6914$ \\
\hline $\mathrm{Y}$ & 1,8042 & 1,6089 & 0,9101 & 1,1054 \\
\hline $\mathrm{X}$ & 0,4055 & 1,0065 & $-0,7060$ & $-0,1049$ \\
\hline $\mathrm{Y}$ & 0,7973 & 0,9926 & $-0,8950$ & $-0,6997$ \\
\hline $\mathrm{X}$ & $-0,0904$ & 0,5107 & $-0,7967$ & $-0,1956$ \\
\hline $\mathrm{Y}$ & 1,3607 & 1,4960 & 0,4067 & 0,6020 \\
\hline $\mathrm{X}$ & 0,4961 & 1,0972 & $-0,2102$ & 0,3909 \\
\hline $\mathrm{Y}$ & 0,5044 & $-0,3091$ & $-1,3984$ & $-1,2031$ \\
\hline
\end{tabular}

A continuación se encuentra el código cíclico óptimo para una canal de transmisión/recepción con línea eléctrica de bajo voltaje. Los códigos cíclicos forman una parte importante de una subclase del código lineal. Si las componentes de $n$-uplas [19] y [20]:

$$
\mathbf{v}=\left[v_{0}, v_{1}, v_{2}, v_{3}, \ldots \ldots . . v_{n-1}\right]
$$

Son cíclicamente desplazadas un lugar a la derecha se obtiene otra $n$-uplas:

$$
\mathbf{v}=\left[v_{n-1}, v_{0}, v_{1}, v_{2}, v_{3}, \ldots \ldots . v_{n-2}\right]
$$

Lo que se denomina un desplazamiento cíclico de $\boldsymbol{v}$. Si las componentes de $\boldsymbol{v}$ son cíclicamente desplazadas $l$ lugares a la derecha, las $n$-uplas resultantes son de la forma:

$$
\mathbf{v}=\left[v_{n-l}, v_{n-l+1}, \ldots \ldots v_{n-1}, v_{0}, v_{1}, \ldots \ldots . . v_{n-l-1}\right]
$$

Claramente $\boldsymbol{v}$ se desplaza cíclicamente en $l$ lugares a la izquierda.

Definición 1: Un código lineal $C$ con $(n, k)$ se denomina código cíclico, si todo desplazamiento cíclico de un vector código en $C$ es también un vector en $C$.

Donde:

$n$ : Largo de la palabra codificada.

$k$ : Largo de la información.

Teorema 1 [20]: El código polinomial no cero de grado mínimo en un código cíclico $C$ es único.

Teorema 2 [20]: Un código cíclico $(n-k)$ tiene un solo código polinomial de grado $(n, k)$ :

$$
g(x)=1+g_{1} x+g_{2} x^{2}+\ldots \ldots+g_{n-k-1} x^{n-k-1}+x^{n-k}
$$

Teorema 3 [20]: El generador polinomial $g(x)$ de un código cíclico $(n, k)$ es un factor de $\left(x^{n}+1\right)$.

Teorema 4 [20]: Si $g(x)$ es un polinomio de grado $(n-k)$ y es un factor de $\left(x^{n}+1\right)$, entonces $g(x)$ genera un código cíclico $(n, k)$.

El proceso de codificación sistemático de un código cíclico tiene los siguientes pasos:

\section{Paso 1:}

Premultiplique el mensaje $u(x)$ por $x^{n-k}$.

\section{Paso 2:}

Obtenga el resto $b(x)$ (los dígitos de chequeo de paridad), desde la división de $x^{n-k} u(x)$ por el generador $g(x)$.

Paso 3:

Combine $b(x)$ y $x^{n-k} u(x)$, para obtener el código polinomial:

$$
c(x)=b(x)+x^{n-k} u(x)
$$


Para un código cíclico sistemático el síndrome se calcula fácilmente como se indica a continuación. El vector que se recibe $\boldsymbol{r}$ se trata como un polinomio de grado $(n-1)$ o menor.

$$
r(x)=r_{0}+r_{1} x+r_{2} x^{2}+\ldots \ldots \ldots+r_{n-1} x^{n-1}
$$

Se divide $r(x)$ por el generador $g(x)$ se obtiene:

$$
r(x)=a(x) g(x)+s(x)
$$

El resto $s(x)$ es un polinomio de grado $(n-k-1)$ o menor. El coeficiente $(n-k)$ de $s(x)$ es el síndrome $s$.

Por la estructura del código cíclico el síndrome $s(x)$ tiene la siguiente propiedad:

Teorema 5 [19]: Sea $s(x)$ el síndrome de un polinomio que se recibe $r(x)=r_{0}+r_{1} x+r_{2} x^{2}+\ldots \ldots \ldots+r_{n-1} x^{n-1}$, entonces el resto $s^{1}(x)$ que resulta de la división $x s(x)$ por el generador polinomial $g(x)$ es el síndrome de $r^{1}(x)$, el cual es un desplazamiento cíclico de $r(x)$.

Teorema 6 [19]: Un código cíclico $(n-k)$ detecta cualquier error de ráfaga de longitud $(n-k)$ o menor.

Teorema 7 [19]: La fracción de error de ráfaga que no se detecta de longitud $(n-k+1)$ es $2^{-(n-k+1)}$.

La decodificación de un código cíclico consiste de los mismos tres pasos como para la decodificación de códigos lineales.

- Cálculo del síndrome.

- Asociación del síndrome a un patrón de error.

- Corrección de error (se usa el algoritmo de Meggit en este artículo).
Los códigos cíclicos óptimos se construyen con la restricción de encontrar el mayor $d_{\min }$, dada la longitud y velocidad, donde $d_{\min }$ es la mínima distancia entre palabra. Existen varios algoritmos para evaluar $d_{\text {min }}$ de un código cíclico [21], [22]. El algoritmo que se usa en este artículo para encontrar el código cíclico óptimo tiene la siguiente estructura: $\operatorname{Sea} \boldsymbol{G}$ una matriz generadora de un código cíclico sistemático, $\boldsymbol{u}$ un vector de longitud $k$ en un campo $\boldsymbol{G} \boldsymbol{F}(2)$ y $w(\boldsymbol{u})$ es el peso del vector $\boldsymbol{u}$. Como $\boldsymbol{G}$ es sistemática, una palabra $c$ se escribe de la forma $c=(u / p)$, donde $p$ es el vector de paridad de longitud $(n-k)$. Para todas $w=1,2, \ldots, k$ que enumera todas las palabras códigos que satisfacen $v(u)=w$, se toman todas las posibles sumas de la fila w en $\boldsymbol{G}$. En cada etapa el peso mínimo de todas las palabras códigos $\boldsymbol{c}$ enumera una cota superior $d_{\min }$, que se denota $d_{c p}$, la cota superior $d_{c p}$ es igual a $\left(w+1 \frac{n}{k}\right)$, donde $[r]$ es el entero más pequeño mayor o igual a $r$. El algoritmo termina cuando $d_{d v} \geq d_{c p}$, donde $d_{d v}$ es la distancia mínima verdadera del código cíclico.

Se resume el algoritmo que se emplea en este artículo en el siguiente lema: Sea $c$ una palabra código de peso $w$ en un código cíclico $(n, k)$ y $\left[\frac{k w}{n}\right]$. Entonces existe un desplazamiento cíclico de $c$ con exactamente $r$ no cero coordenadas entre las primeras $k$ coordenadas.

El esquema que se propone para mejorar la confiabilidad del sistema de transmisión/recepción para canales con ruido clase $\mathrm{A}$, se muestra en la Figura 2.

De acuerdo con la Figura 2, se tiene el codificador de código cíclico $c(t)$, el que le suma redundancia

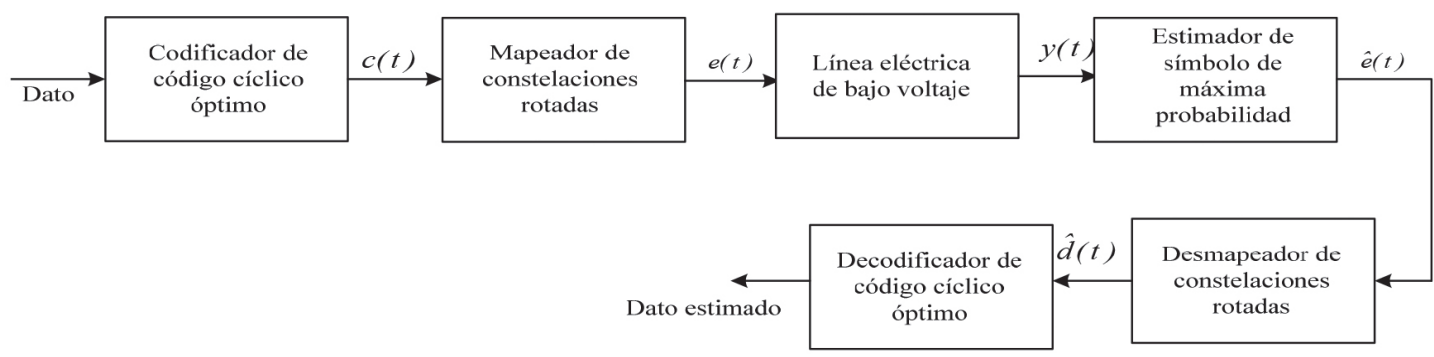

Figura 2. Esquema que se propone para mejorar la confiabilidad en un sistema de transmisión/recepción para canales con ruido clase A. 
a los bits que se transmiten, le sigue el mapeador de constelaciones rotadas $e(t)$, que toma dos bits y la convierte en un símbolo de la constelación de cuatro símbolos con rotación de ángulos (circular, rectangular y hexagonal) o toma cuatro bits y la convierte en un símbolo de la constelación de dieciséis símbolos con rotación de ángulos (circular, rectangular y hexagonal).

A la señal que se transmite se le suma el ruido, efecto que provoca el canal de transmisión (línea eléctrica de bajo voltaje) $y(t)$. En el receptor se tiene el estimador de símbolo de máxima probabilidad, cuya función es estimar el símbolo que se transmite $\hat{e}(t)$, el desmapeador de constelaciones $\hat{d}(t)$, realiza la función inversa del mapeador, por último se tiene al decodificador de código cíclico que corrige los bits que llegaron erróneos.

\section{RESULTADOS Y SU DISCUSIÓN}

Los parámetros del ruido en la línea eléctrica de bajo voltaje son: valor del índice impulsivo $A=0,01$ y la razón de ruido impulsivo $\Gamma=0,001$, con estos valores el ruido es altamente impulsivo como se muestra en la Figura 3.

El código cíclico óptimo que se encuentra en este artículo tiene las siguientes características: largo de la palabra $n=50$, el número de bit de paridad $r=16$, el largo del mensaje $k=34$. Este código puede corregir hasta $b=8$ bits consecutivos y 11 bits erróneos. El $r$ se determina como: $r=2 b$.
El polinomio mínimo generador del código es:

$$
g(x)=1+x^{3}+x^{4}+x^{6}+x+x^{11}+x^{13}+x^{16}
$$

Este código tiene una velocidad de $R=\frac{k}{n}=\frac{34}{50}=0,68$.

La Figura 4 muestra el desempeño del BER versus SNR para la combinación de código cíclico óptimo y constelaciones rotadas de cuatro símbolos.

La Figura 5 muestra el desempeño del BER versus SNR para la combinación de código cíclico óptimo y constelaciones rotadas de dieciséis símbolos.

De acuerdo con la Figura 3, con los parámetros dados se observa que el ruido tiene una característica altamente impulsiva.

De la Figura 4, donde se combinan códigos cíclicos óptimos con constelaciones rotadas (rectangular, circular y hexagonal) de cuatro símbolos se obtiene la disminución del BER en un orden de magnitud de por lo menos de $1 \times 10^{-2}$ para un SNR de 15 $\mathrm{dB}$ con relación a cuando no se utiliza el código cíclico óptimo. De la Figura 5, donde se combinan códigos cíclicos óptimos con constelaciones rotadas (rectangular, circular y hexagonal) de dieciséis símbolos se obtiene la disminución del BER en un orden de magnitud de por lo menos de $1 \times 10^{-2}$ para un SNR de 15 dB en comparación a cuando no usa el código cíclico óptimo.

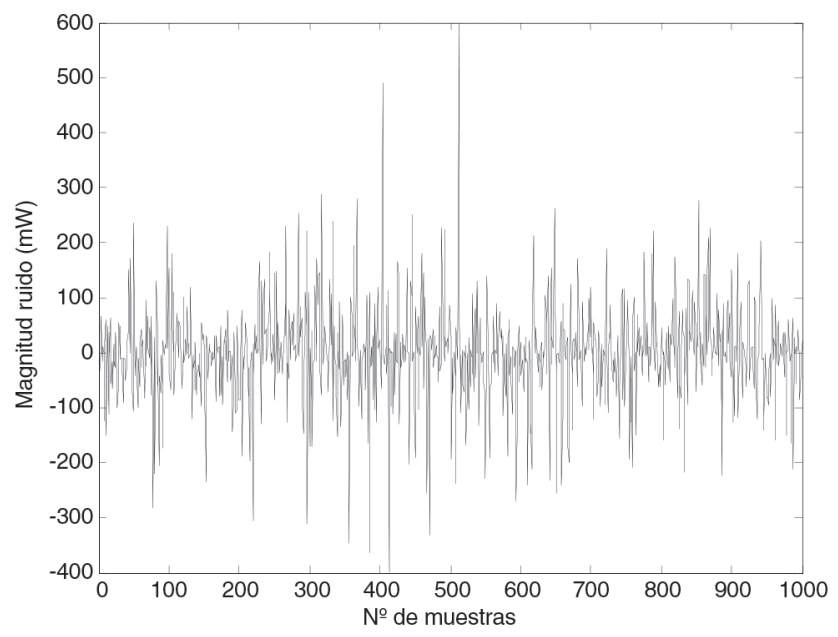

Figura 3. Característica del ruido presente en la línea eléctrica de bajo voltaje. 


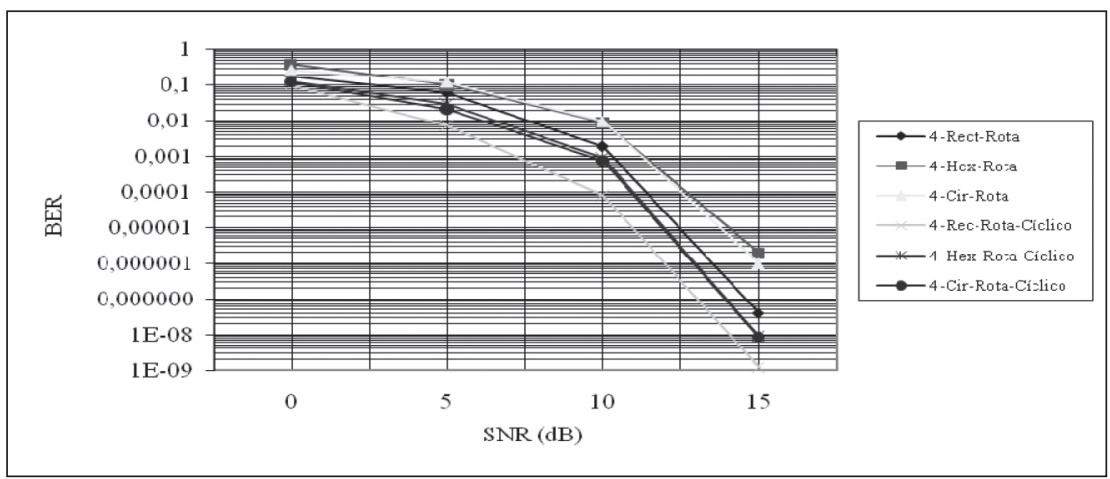

Figura 4. Desempeño del BER versus SNR para la combinación de código cíclico óptimo y constelaciones rotadas de cuatro símbolos.

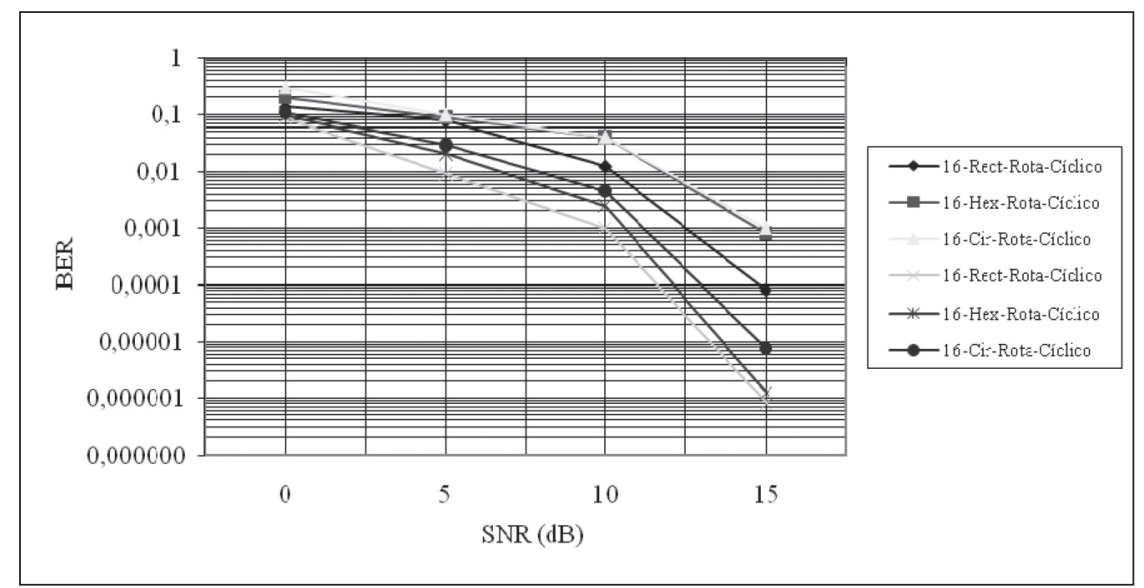

Figura 5. Desempeño del BER versus SNR para la combinación de código cíclico óptimo y constelaciones rotadas de dieciséis símbolos.

De las Figuras 4 y 5 se infiere que las rotaciones de ángulos de las constelaciones rectangular, circular y hexagonal de cuatro y dieciséis símbolos disminuye la probabilidad de error de bit, porque los ángulos de rotaciones óptimos para las constelaciones bajo estudio permiten encontrar las mayores distancias entre los símbolos, lo que a su vez permite que el detector de símbolos de máxima probabilidad aumente la probabilidad de detectar el símbolo en forma correcta.

El desempeño del código cíclico óptimo es bueno, lo que se observa de las gráficas de las Figuras 4 y 5 porque baja notablemente la probabilidad de error de bits cuando se compara el desempeño sin utilizar el código cíclico óptimo, la razón de esto porque el código propuesto en este artículo permite detectar y corregir hasta ocho bits consecutivos (de ráfaga) característico de un canal de línea eléctrica de bajo voltaje (ruido impulsivo).

\section{CONCLUSIONES}

De acuerdo con los resultados que se obtienen se puede inferir que la combinación de código cíclico óptimo y las constelaciones rotadas rectangular, circular y hexagonal de cuatro y dieciséis símbolos logran un muy buen desempeño en un canal de transmisión constituido por las líneas eléctricas de bajo voltaje, aumentando la confiabilidad del sistema de transmisión/recepción al disminuir la razón de error de bits. La razón de esto es que la rotación de las constelaciones rectangular, circular y hexagonal cuatro y dieciséis símbolos aumenta la región de decisión del detector de máxima probabilidad, porque los ángulos de rotación 
maximizan las distancias entre los símbolos de las constelaciones. El código cíclico óptimo que es propuesto en este artículo maximiza la distancia mínima de las palabras del código, lo que se refleja en la disminución del error de bits cuando se compara con el caso en que solo se utilizan las constelaciones rotadas.

\section{AGRADECIMIENTOS}

El autor agradece a los revisores anónimos por sus comentarios y sugerencias que mejoraron este artículo y a CONICYT por patrocinar este trabajo mediante el proyecto CONICYT FONDEF IDeA CA13I10318.

\section{REFERENCIAS}

[1] W. Fernández R. "Rotación óptima de las constelaciones hexagonal, circular y rectangular para canales con ruido clase A". En proceso de publicación el año 2014. IEEE Latin América Transactions.

[2] M.K. Simon and J.G. Smith. "Hexagonal multiple phase and amplitude shift keyed signal set". IEEE Transactions on Communications. Vol. 21, Issue 10, pp. 11081115. October, 1973.

[3] L. Leszek Szczecinski and M. Bacic. "Constellations design for multiple transmissions: Maximizing the minimum squared Euclidean distance”. IEEE Wireless Communications and Networking Conference. Vol. 2, pp. 1066-1071. March, 2005.

[4] G.D. Forney Jr. and L.-F. Wei. "Multidimensional constellations-Part I: Introduction, figures of merit, and generalized cross". IEEE Journal on Selected Areas in Communications. Vol. 7, Issue 6, pp. 877892. August, 1989.

[5] A.D. Spaulding and D. Middleton. "Optimum reception in an impulsive interference environment. Part I: Coherent detection". IEEE Transactions Communications. Vol. 25, Issue 9, pp. 910-922. September, 1977.

[6] A.D. Spaulding and D. Middleton. "Optimum reception in an impulsive interference environment. Part II: Incoherent detection". IEEE Transactions on Communications.
Vol. 25, Issue 9, pp. 924-934. September, 1977.

[7] V.P. Semerenko. "Burst-error correction for cyclic codes". IEEE EUROCON 2009, pp. 1650-1655. 19-23 May, 2009.

[8] E. Gorog. "Some new classes of cyclic codes used for burst-error correction". IBM Journal of Research and Development, pp. 102-111. April, 1963.

[9] C. Koscielny. "Constructing a better cyclic code than cyclic Reed-Solomon code". IEEE Transactions on Information Theory. Vol. 41, Issue 3, pp. 1191-1194. July, 1995.

[10] C.L. Chen. "On a $(145,32)$ binary cyclic code". IEEE Transactions on Information Theory. Vol. 45, Issue 7, pp. 2546-2547. November, 1999.

[11] S. Song, K. Abdel-Ghaffar, Z. Ding, W. Fong and M.P. Fassorier. "Burst decoding of cyclic codes based on circulant parity check matrices". IEEE Transactions on Information theory. Vol. 50, Issue 3, pp. 1038-1047. March, 2010.

[12] C. Ding. "The weigth distribution of some irreducible cyclic codes". IEEE Transactions on Information Theory. Vol. 55, Issue 3, pp. 955-960. March, 2009.

[13] D. Augost and M. Bardet. "On formula for decoding binary cyclic codes". ISIT 2007, Nice France, pp. 2646-2650. June 29, 2007.

[14] J. Luo, TY. Tang and H. Wang. "Cyclic codes and sequences generalized Kasami case". IEEE Transactions on Information Theory. Vol. 56, Issue 5, pp. 2130-2142. May, 2010.

[15] C.M. Tomlinson, M. Grassi, R. Horan, M. Ahmed and M. Ambroze. "New linear codes derived from binary cyclic codes of length 151". IEE Proceedings Communication. Vol. 153, Issue 5, pp. 581-585. October, 2006.

[16] G. Vega. "A note about two weight non irreducible cyclic code". IEEE Transactions on Information Theory. Vol. 58, Issue 4, pp. 2263-2364. April, 2012.

[17] F. Ramy, M. Rabab and H. Salwa. "A novel high speed encoder for long cyclic codes". IEEE Communications Letters. Vol. 17, Issue 5, pp. 984-987. May, 2013.

[18] Sh. Lin and D.J. Costello. "Error control coding: Fundamentals and Application". Prentice Hall. 1983. 
[19] H. Cohen. "A course in computational algebraic number theory". Third Edition. Springer. 1996.

[20] W. Peterson. "Error correcting codes". MIT Press. Cambridge, Massachusetts. Second Edition. 1962.
[21] C.L. Chen. "Some results on algebraically structured error-correcting codes". PH. D. dissertation. University of Hawaii. 1969.

[22] J.F. Voloch. "Computing the minimal distance of cyclic codes". Computational and Applied Mathematics. Vol. 24, Issue 3, pp. 393-398. 2005. 